\title{
Slow drift compensation in long-term measurements in fluorescence nanoscopy with blinking colloidal semiconductor quantum dots
}

\author{
Aleksei Baev* and Aleksei Rozhentsov \\ Volga State University of Technology, Yoshkar-Ola 420000, Russia
}

\begin{abstract}
In this work we present an approach for image processing accuracy increasing in fluorescence nanoscopy of single colloidal quantum dots with blinking fluorescent behavior. The approach is based on the estimation of parameters, describing the slow collective drift, according to the information about a long series of frames with fluorescent images of single blinking quantum dots. The results of collective drift compensation for single colloidal quantum dots $\mathrm{CdSe} / \mathrm{ZnS}$ supper-resolution image are demonstrated.
\end{abstract}

The presence of a slow drift - spatial image shift in time - is one of the serious problems of various nanoscopy techniques. The characteristic values of the shifts can vary from tens to several hundred or more nanometers per hour. The potential reasons are instabilities in the sample positioning system, slow changes in the spatial position of the optical elements in the recording system associated with mechanical and temperature instabilities. As a result, the supper-resolution images of particles are "blurred", which leads to a decrease in the accuracy of the estimation of their parameters. In this paper, we propose an approach that reduces the effects of slow drift in long-term measurements in experiments on luminescent nanoscale with single flickering quantum dots. The approach is based on estimating the parameters of a function describing the collective drift and sub-pixel shift compensating in each CCD-camera frame in image post-processing.

In present time there exist a few approaches to compensate nanodrifts: by measuring the interframe shift based on calculating the cross-correlation function between frames [1], by using the special embedded markers [2], etc. In this paper, we propose an approach based on the use of information on the subdi-fractional coordinates of flickering quantum dots recorded in a sufficiently long series of frames (from several hundred to several thousand frames) that does not require special markers. This approach makes it possible to obtain drift-corrected superresolution images of single quantum dots without additional markers. To do this, information about the coordinates of all the QDs found in the series of frames under study is grouped into a common data array by subtracting the mathematical expectation of the coordinates of the QDs within the track from the coordinates of each QD. The set of points thus obtained is approximated by a polynomial of a given degree. The obtained functional dependence will characterize the law of global drift of all marks.

*Corresponding author: kruzenshteyn@yandex.ru 
To show the possibilities of suggested approach we use the experimental data on fluorescence images of single colloidal QDs CdSe/ZnS (with diameter of emitting core $\sim 4$ $\mathrm{nm}$ ) kindly provided by the Single-molecule research team from the Institute for Spectroscopy of the Russian Academy of Sciences [3-5]. The fluorescence of single QD were excited by CW laser (Coherent Verdi V6) on the wavelength $532 \mathrm{~nm}$ with excitation intensity $\sim 100 \mathrm{~W} / \mathrm{cm}^{2}$. The fluorescence images were detected by EM CCD camera (Luca Andor) with exposure time $100 \mathrm{~ms}$ per frame.

Figure 1 demonstrates the dependence of the change in the subdiffractional coordinate $Y$ for several single QDs and corresponding functions which describe observed global drifts for the coordinates $\mathrm{X}$ (red line) and Y (green line).
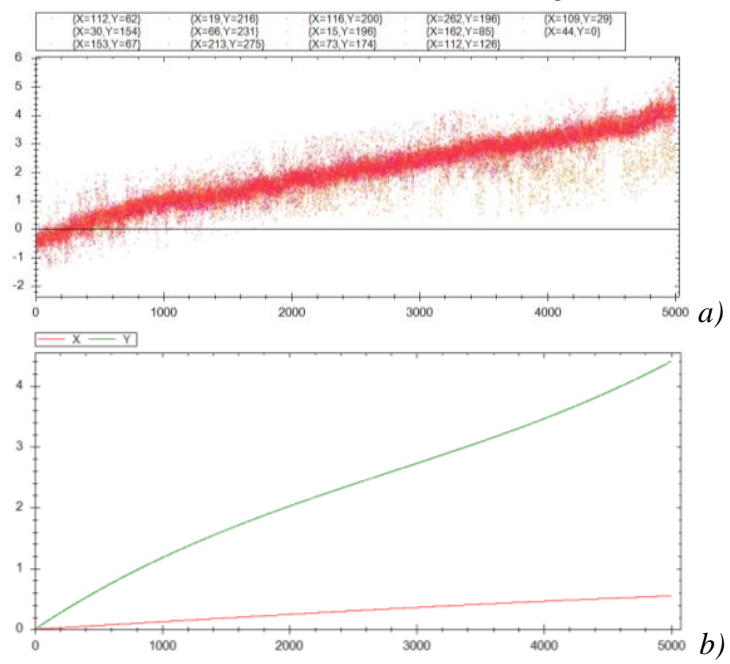

Fig. 1. Dependence of the change in the subdifraction coordinate $\mathrm{Y}$ for several quantum dots (a). The result of calculating the global drift for the coordinates $\mathrm{X}$ (red graph) and $\mathrm{Y}$ (blue graph) (b). The abscissa is the frame number.

The Figure 2 (a) shows an example of the "superresolution" image of single QD subjected to the slow drift. This superresolution image is constructed from restored (from each CCD-frame) QD coordinates as 2D-histogram of coordinates distribution. The significant drift along the $\mathrm{Y}$ coordinate is clearly observed. The "superresolution" image is elongated along the drift direction.

Figure $2 b$ shows the image obtained for the same series of frames, after compensation for global drift. The density of the restored coordinates is a symmetrical figure, i.e., a circle of the radius equal to the accuracy of coordinates determination in a single measurement. Thus in this paper we show that slow drifts can be compensated by extended measurements and correct polynomial analysis.

The work was supported by the Russian Science Foundation (grant No. 17-72-20266).

\section{References}

1. M. J. Mlodzianoski et al., Opt. Express, 19, 15009 (2011)

2. H. Ma et al, Biophysical Journal, 112, 2196 (2017)

3. A. V. Naumov, I. Y. Eremchev, A. A. Gorshelev, EPJ D 68, 348 (2014)

4. A. L. Shchukina, I. Y. Eremchev, A. V. Naumov, Phys. Rev. E, 92, 032102 (2015)

5. I. Y. Eremchev, I. S. Osad'ko, A. V. Naumov, J. Phys. Chem. C, 120, 22004 (2016) 